\title{
Zero-Mass Infinite Spin Representations of the Poincaré Group and Quantum Field Theory
}

\author{
JAKOB YNGVASON \\ Institut für Theoretische Physik, Universität Göttingen
}

Received December 15, 1969

\begin{abstract}
It is shown that a local quantized field with a manifestly covariant transformation law under the Poincaré group cannot have nonvanishing matrix elements between the vacuum and an irreducible subspace of zero mass and infinite spin.
\end{abstract}

\section{Introduction}

The zero-mass infinite spin representations of the Poincaré group $\mathscr{P}_{+}^{+}[1-3]$ do not seem to correspond to anything in nature and have consequently received little attention from physicists. Nevertheless, it might be instructive to know whether these "strange" representations violate some fundamental principle, or if their exclusion from physical theories is an independent postulate. The present paper deals with the question whether they can appear in a local quantum field theory. This seems to be a natural question since at least free fields can be constructed corresponding to any of the other irreducible representations of $\mathscr{P}_{+}^{t}$ that satisfy the spectrum condition [4]. It is however clear, that if we want to extend this construction to the case of infinite spin, we must allow infinite dimensional representations of $S L(2, \mathbb{C})$ in the transformation law of the field. We modify the usual Wightman axioms [5] in accordance with this fact.

It turns out, however, that this modification is not sufficient. The generalized Wightman axioms, especially local commutativity and the local (manifestly covariant) transformation law, will be shown to exclude the "strange" representations in the following sense: The field operators cannot have nonvanishing matrix elements between the vacuum and states that transform according to an irreducible representation of zero mass and infinite spin. In particular, there are no free fields corresponding to these representations.

14 Commun. math. Phys., Vol. 18 


\section{The Zero-Mass Infinite Spin Representations of $\mathscr{P}_{+}^{\dagger}$}

$\mathscr{P}_{+}^{\dagger}$ is the semi-direct product of the translation group $\mathbb{R}^{4}$ and $S L(2, \mathbb{C})^{1}$. The irreducible representations of interest here are defined as follows $[1,3]$ :

Let $M_{0}^{+}$be the mantle of the forward light cone and $\left\{\Lambda_{p}\right\}$ a family of Lorentz transformations with $\Lambda_{p} p=(1,0,0,1)=\bar{p} \in M_{0}^{+}$for all $p \in M_{0}^{+}$. $\Lambda_{p}$ should depend continuously on $p$. The transformation $K(p, \Lambda)$ $=\Lambda_{p} \cdot \Lambda \cdot \Lambda_{\Lambda}^{-1}{ }^{-1}$ leaves $\bar{p}$ invariant for all $p \in M_{0}^{+}$and $\Lambda \in S L(2, \mathbb{C})$ and is thus an element of the "little group" of $\bar{p}$. This group is isomorphic to the covering group of the two dimensional Euclidean group and will be denoted by $\mathscr{E}_{2}$. It consists of matrices of the form

$$
\Lambda_{z, \varphi}=\left(\begin{array}{ll}
1 & z \\
0 & 1
\end{array}\right) \cdot\left(\begin{array}{ll}
e^{i \varphi / 2} & 0 \\
0 & e^{-i \varphi / 2}
\end{array}\right), \quad z \in \mathbb{C}, \varphi \in \mathbb{R} .
$$

Let $H$ be the Hilbert space of complex valued $L_{2}$-functions on the interval $[0,2 \pi]$. It is convenient to think of these functions as periodic with period $2 \pi$. For each $\Xi$ and $\alpha$ with $0<\Xi<\infty, \alpha=0$ or $1 / 2$, there is an irreducible unitary representation $V_{\varepsilon, \alpha}$ of $\mathscr{E}_{2}$ on $H$ defined by

$$
\left(V_{\Xi, \alpha}\left(\Lambda_{z, \varphi}\right) f\right)(\theta)=\{\exp i(\Xi|z| \cos (\arg z-\theta)+\alpha \varphi)\} f(\theta-\varphi) .
$$

$V_{\Xi, \alpha}$ induces an irreducible unitary representation $U_{\Xi, \alpha}$ of $\mathscr{P}_{+}^{\uparrow}$ on the Hilbert space

$$
\mathscr{H}=\int^{\oplus} H \theta\left(p^{0}\right) \delta\left(p^{2}\right) d^{4} p
$$

defined by

$$
\left(U_{\xi, \alpha}(a, \Lambda) \Phi\right)(p)=e^{i p \cdot a} V_{\Xi, \alpha}(K(p, \Lambda)) \Phi\left(\Lambda^{-1} p\right)
$$

for $a \in \mathbb{R}^{4}, \Lambda \in S L(2, \mathbb{C}), \Phi \in \mathscr{H}$. The invariant scalar product is $p \cdot a$ $=p^{0} a^{0}-p^{1} a^{1}-p^{2} a^{2}-p^{3} a^{3}$.

In contrast to the finite spin case, the transformations $\Lambda_{z, 0}$ are here not represented by the identity. A consequence of this is the following lemma, which turns out to be important for our proof:

Lemma 1. Let $\Lambda_{\lambda}$ denote the matrix $\left(\begin{array}{ll}\lambda & 0 \\ 0 & \lambda^{-1}\end{array}\right) \in S L(2, \mathbb{C}), \lambda \in \mathbb{R}-\{0\}$. Then $\Lambda \mapsto \Lambda_{\lambda} \cdot \Lambda \cdot \Lambda_{\lambda}^{-1}$ is an automorphism of $\mathscr{E}_{2}$. Moreover, the representation $\Lambda \mapsto V_{\Xi, \alpha}\left(\Lambda_{\lambda} \cdot \Lambda \cdot \Lambda_{\lambda}^{-1}\right)$ is equivalent to $V_{\lambda^{2} \Xi, \alpha}$, and thus not equivalent to $V_{\varepsilon, \alpha}$.

The lemma follows immediately from the fact that $\Lambda_{\lambda} \cdot \Lambda_{z, \varphi} \cdot \Lambda_{\lambda}^{-1}$ $=\Lambda_{\lambda^{2} z, \varphi}$. Insertion of this into (1) proves the statement.

${ }^{1}$ We assume the same correspondence between matrices in $S L(2, \mathbb{C})$ and Lorentz transformations as in [5]. $S L(2, \mathbb{C})$ operates on $\mathbb{R}^{4}$ according to this correspondence. 
The 1-parameter group $\left\{\Lambda_{0, \varphi} \mid \varphi \in \mathbb{R}\right\}$ is the group of rotations about the $x_{3}$-axis. Its infinitesimal generator represents the spin component along this direction. In the representations $V_{z, \alpha}$ the spectrum of this operator is unbounded, the eigenvalues being $0, \pm 1, \pm 2, \ldots$ for $\alpha=0$ and $\pm 1 / 2, \pm 3 / 2, \ldots$ for $\alpha=1 / 2$. Hence the notation "infinite spin".

The geometrical meaning of the transformations $\Lambda_{z, 0}$ is perhaps most easily seen if we write the corresponding Lorentz transformation as the product of a rotation and a pure Lorentz transformation. Let $R(\theta)$ denote a rotation about the $x_{2}$-axis with rotation angle $\theta$ counted positive from the $x_{1}$-direction to the $x_{3}$-direction. Put $L(\chi, \varphi)=R(\varphi)$ - $L(\chi) R(\varphi)^{-1}$ where $L(\chi)$ is a pure Lorentz transformation in the $x_{1}$-direction of velocity $v / c=\operatorname{Artanh} \chi$. It is sufficient to consider the case $z \in \mathbb{R}$ and in that case

$$
\Lambda_{z, 0}=R(-2 \varphi) L(\chi, \varphi)
$$

where the parameters $\chi$ and $\varphi$ are connected to each other and to $z$ by the equations

$$
\begin{aligned}
& \sinh \chi=2 \frac{\sin \varphi}{\cos ^{2} \varphi} \\
& \tan \varphi=z / 2 .
\end{aligned}
$$

\section{Assumptions and Results}

As mentioned in the introduction we will use slightly generalized Wightman axioms:

1. The field operators $A(\sigma, f)$ are defined for each test function $f \in \mathscr{D}$ and each $\sigma \in K$, where $K$ is some linear space. The operators $A(\sigma, f)$ and their adjoints map a dense subset $\mathscr{H}_{D}$ of a separable ${ }^{2}$ Hilbert space $\mathscr{H}$ into itself. They depend linearly on $f$ and $\sigma$, and without loss of generality we may assume the existence of an antilinear operator on $K, \sigma \mapsto \sigma^{*}$, such that $A(\sigma, f)^{*}=A\left(\sigma^{*}, \bar{f}\right)$, where $A(\sigma, f)^{*}$ is the adjoint operator and $\bar{f}$ the complex conjugate of $f$. The matrix elements $(\Phi, A(\sigma, f) \Psi)$ with $\Phi, \Psi \in \mathscr{H}_{D}$ are continuous in $f$, i.e. they are distributions.

2. $\mathscr{H}$ carries a continuous unitary representation $U$ of $\mathscr{P}_{+}^{\dagger} . U$ satisfies the spectrum condition: $U(a, 1)=\int e^{i p \cdot a} d E_{p}$ with supp $d E_{p} \subset \bar{V}^{+}$(forward light cone); the operators $U(a, \Lambda)$ leave $\mathscr{H}_{D}$ invariant, and there is a unique invariant vacuum state $\Omega \in \mathscr{H}_{D}$.

${ }^{2}$ This assumption is in fact redundant. Using the separability of $\mathscr{D}$ and $S L(2, \mathbb{C})$ and the continuity postulates, it is not difficult to prove that if an irreducible representation appears in a field theory at all, then it also appears in a field theory on a separable Hilbert space. 
3. Local commutativity (or anti-commutativity):

$$
[A(\sigma, x) A(\varrho, y)]_{\mp}=0 \text { for }(x-y)^{2}<0, \sigma, \varrho \in K .
$$

4. Transformation law: There is a representation $D$ of $S L(2, \mathbb{C})$ on $K$ such that

$$
U(a, \Lambda) A(\sigma, x) U(a, \Lambda)^{-1}=A(D(\Lambda) \sigma, \Lambda x+a) .
$$

5. Polynomials in the field operators generate a dense set in $\mathscr{H}$ when applied to the vacuum.

The last postulate is not significant since we are anyway only going to consider vectors that are obtained by applying one field operator to the vacuum. Let $\mathscr{H}^{\prime}$ denote the subspace of such vectors:

$$
\mathscr{H}^{\prime}=\overline{\left\{\sum_{i=1}^{n} A\left(\sigma_{i}, f_{i}\right) \Omega \mid n<\infty, \sigma_{i} \in K, f_{i} \in \mathscr{D}\right\}} .
$$

(The bar denotes closure in the topology of $\mathscr{H}$.) We intend to prove the following theorem:

Theorem. The Hilbert space of a field theory satisfying axioms 1-4 contains no subspace $\mathscr{H}_{\text {irr }}$ such that:

i) $\mathscr{H}_{\text {irr }}$ is invariant under $U(a, \Lambda)$ and the restriction $U \mid \mathscr{H}_{\text {irr }}$ is an irreducible representation of zero mass and infinite spin.

ii) $E_{\mathrm{irr}} \mathscr{H}^{\prime} \neq\{0\}$, where $E_{\mathrm{irr}}$ denotes the projector on $\mathscr{H}_{\mathrm{irr}}$.

The proof can be outlined as follows:

If $U_{\xi, \alpha}$ is to appear as a subrepresentation of $U$, then the representation $D$ of $S L(2, \mathbb{C})$ must contain $V_{\Xi, \alpha}$ when restricted to $\mathscr{E}_{2}$. Since $D$ is not only defined on $\mathscr{E}_{2}$, but on $\Lambda_{\lambda}$ as well, it is implied by Lemma 1 that this restriction also contains all other representations $V_{\lambda^{2} \Xi, \alpha}, \lambda \neq 0$. Which of these representations appears in $U$ is determined by the two-point function. The structure of the latter implied by analyticity in the extended tube, which in turn is a consequence of the spectrum condition and locality, is too restrictive to "filter out" one of these uncountably many representations. We begin with an investigation of this structure.

\section{Structure of the Two-Point Function}

The representation of the translation group has the spectral decomposition $U(a, 1)=\int e^{i p \cdot a} d E_{p}$. The support of $d E_{p}$ is a Lorentz invariant subset of $\bar{V}^{+}$. We assume that $U$ has zero mass components and decompose $d E_{p}$ into three parts

$$
d E_{p}=E_{0} \delta(p)+d E_{p}^{1}+d E_{p}^{2}
$$


where $E_{0}$ is the projector on $\Omega, \operatorname{supp} d E_{p}^{1}=M_{0}^{+}$such that $\{0\}$ is a null set for $d E_{p}^{1}$ and $M_{0}^{+}$is a null set for $d E_{p}^{2}$. This decomposition is evidently unique. We put $E_{1}=\int d E_{p}^{1}$ and $E_{2}=\int d E_{p}^{2}$. These projectors commute with all $U(a, \Lambda)$.

The object of interest is the distribution

$$
W_{\sigma \varrho}^{1}(x-y)=\left(\Omega, A\left(\sigma^{*}, x\right) E^{1} A(\varrho, y) \Omega\right) .
$$

Its structure is described by the following lemma:

Lemma 2. The Fourier transform

can be written as

$$
\tilde{W}_{\sigma \varrho}^{1}(p)=\int e^{i p \cdot \xi} W_{\sigma \varrho}^{1}(\xi) d \xi
$$

$$
\tilde{W}_{\sigma \varrho}^{1}(p)=M_{\sigma \varrho}(p) \theta\left(p^{0}\right) \delta\left(p^{2}\right)
$$

where $M_{\sigma \varrho}(p)$ is a polynomial in $p$ for all $\sigma, \varrho \in K . M_{\sigma \varrho}(p)$ has the following properties for $p \in M_{0}^{+}$:

i) $M_{\sigma \varrho}(p)$ defines a positive semidefinite sesquilinearform on $K$, i.e. $M_{\sigma \varrho}(p)$ is linear in $\varrho$, antilinear in $\sigma, M_{\sigma \varrho}(p)=\overline{M_{\varrho \sigma}(p)}$ and $M_{\sigma \sigma}(p) \geqq 0$.

ii) $M_{D(\Lambda) \sigma, D(\Lambda) \varrho}(\Lambda p)=M_{\sigma \varrho}(p)$ and $M_{\sigma, D(\Lambda) \varrho}(p)$ is continuous in $\Lambda \in S L(2, \mathbb{C})$.

Proof. The two-point function $W_{\sigma \varrho}(x-y)=\left(\Omega, A\left(\sigma^{*}, x\right) A(\varrho, y) \Omega\right)$ is a tempered distribution because of positivity and according to [6] it is the boundary value of an analytic function

$$
W_{\sigma \varrho}(\zeta)=\sum_{i=1}^{n_{\sigma} \varrho} P_{\sigma \varrho}^{i}(\zeta) F_{\sigma \varrho}^{i}\left(\zeta^{2}\right)
$$

where the $P_{\sigma e}^{i}$ are polynomials and the $F_{\sigma \varrho}^{i}$ are analytic in the cut plane $\mathbb{C}-\mathbb{R}^{+}$. By the method of Lemma 1 in [7] it is then easy to prove that the Fourier transform $\tilde{W}_{\sigma \varrho}$ is of the form

$$
\tilde{W}_{\sigma \varrho}(p)=\sum_{i=1}^{m_{\sigma e}} Q_{\sigma \varrho}^{i}(p) G_{\sigma \varrho}^{i}(p)
$$

with polynomials $Q_{\sigma \varrho}^{i}$ and Lorentz invariant distributions $G_{\sigma \varrho}^{i}$. Because of positivity and the spectrum condition, the $G_{\sigma \varrho}^{i}$ are measures with support in $\bar{V}^{+}$. Each $G_{\sigma \varrho}^{i}$ can be written uniquely as

$$
G_{\sigma \varrho}^{i}(p)=a_{\sigma \varrho}^{i} \delta(p)+b_{\sigma \varrho}^{i} \theta\left(p^{0}\right) \delta\left(p^{2}\right)+H_{\sigma \varrho}^{i}(p)
$$

where $M_{0}^{+}$is a null set for $H_{\sigma \varrho}^{i}(p)$. The corresponding decomposition of $W_{\sigma \varrho}$ can also be achieved with the projectors $E_{0}, E_{1}$ and $E_{2}$ :

$$
\begin{aligned}
W_{\sigma \varrho}(x-y)= & \left(\Omega, A\left(\sigma^{*}, x\right) E_{0} A(\varrho, y) \Omega\right)+\left(\Omega, A\left(\sigma^{*}, x\right) E_{1} A(\varrho, y) \Omega\right) \\
& +\left(\Omega, A\left(\sigma^{*}, x\right) E_{2} A(\varrho, y) \Omega\right) .
\end{aligned}
$$


Comparison of (3), (4), and (5) then shows that $W_{\sigma e}^{1}$ has the desired form. Property i) follows from the properties of the scalar product in $\mathscr{H}$ and ii) from the transformation law and continuity of the representation $U$.

\section{Reconstruction of $\mathscr{H}_{1}=E_{1} \mathscr{H}^{\prime}$}

$\mathscr{H}_{1}=E_{1} \mathscr{H}^{\prime}$ is the space of zero-mass states in $\mathscr{H}^{\prime}$. It is invariant under $U$ and our aim is to show that the restriction $U \mid \mathscr{H}_{1}=U_{1}$ does not contain a $U_{\Xi, \alpha}$ as a subrepresentation. As a first step, we reconstruct $\mathscr{H}_{1}$ in a concrete form with the aid of $\widetilde{W}_{\sigma \varrho}^{1}$.

Let $\bar{p}$ and $\Lambda_{p}$ be as in Section 2 and $N_{1}=\left\{\sigma \in K \mid M_{\sigma \sigma}(\bar{p})=0\right\}$. Because of Lemma 2 i) we can define a scalar product on the quotient space $K_{1}=K / N_{1}$ :

$$
\left([\sigma]_{1},[\varrho]_{1}\right)=M_{\sigma \varrho}(\bar{p})
$$

where $[\tau]_{1}$ denotes the rest class $\tau+N_{1}$. We complete $K_{1}$ to a Hilbert space $\bar{K}_{1}$ and define a representation $V_{1}$ of $\mathscr{E}_{2}$ on this space by

$$
V_{1}(\Lambda)[\sigma]_{1}=[D(\Lambda) \sigma]_{1} .
$$

By Lemma 2 ii) $V_{1}$ is well defined, unitary and continuous. On the Hilbert space

$$
\widetilde{\mathscr{H}}_{1}=\int^{\oplus} \bar{K}_{1} \theta\left(p^{0}\right) \delta\left(p^{2}\right) d^{4} p
$$

it induces a unitary representation of $\mathscr{P}_{+}^{\dagger}$ :

$$
\begin{array}{r}
\left(\tilde{U}_{1}(a, \Lambda) \Phi\right)(p)=e^{i p \cdot a} V_{1}\left(\Lambda_{p} \cdot \Lambda \cdot \Lambda_{\Lambda^{-1} p}^{-1}\right) \Phi\left(\Lambda^{-1} p\right), \\
a \in \mathbb{R}^{4}, \Lambda \in S L(2, \mathbb{C}), \Phi \in \mathscr{H}_{1} .
\end{array}
$$

It is straightforward to verify that this representation is unitarily equivalent to $U_{1}$. The corresponding isomorphism $\mathscr{H}_{1} \rightarrow \widetilde{\mathscr{H}}_{1}$ orders $E_{1} A(\sigma, f) \Omega$ to the function $\left(p \mapsto \tilde{f}(p)\left[D\left(\Lambda_{p}\right) \sigma\right]_{1}\right) \in \tilde{\mathscr{H}}_{1}$.

\section{Proof of the Theorem}

According to the considerations above, $U_{1}$ is determined by the representation $V_{1}$ of $\mathscr{E}_{2}$. The statement of our theorem is thus equivalent to the statement that none of the representations $V_{\Sigma, \alpha}$ can appear as a subrepresentation of $V_{1}{ }^{3}$. We now proceed to prove this latter statement.

Lemma 3. i) Let $\operatorname{deg} M_{\sigma \varrho}$ denote the degree of the polynomial $M_{\sigma \varrho}(p)$. If $\sigma, \varrho \in K^{(n)}=\left\{\tau \in K \mid \operatorname{deg} M_{\tau \tau} \leqq n\right\}$, then $\operatorname{deg} M_{\sigma \varrho} \leqq n$.

\footnotetext{
subspace.

${ }^{3}$ By subrepresentation we mean the restriction of a representation to an invariant
} 
ii) The spaces $\mathscr{H}_{1}^{(n)}=\overline{\text { Linear span of }\left\{E_{1} A(\sigma, f) \Omega \mid \sigma \in K^{(n)}, f \in \mathscr{D}\right\}}$ are invariant under $U_{1}$, and every irreducible subrepresentation of $U_{1}$ is unitarily equivalent to a subrepresentation of one of the restrictions $U_{1} \mid \mathscr{H}_{1}^{(n)}$.

Proof. The first part of the lemma follows from the Cauchy-Schwarz inequality

$$
\left|M_{\sigma \varrho}(p)\right|^{2} \leqq M_{\sigma \sigma}(p) M_{\varrho \varrho}(p)
$$

for all $p \in M_{0}^{+}$, which implies

$$
\operatorname{deg} M_{\sigma \varrho} \leqq 1 / 2\left(\operatorname{deg} M_{\sigma \sigma}+\operatorname{deg} M_{\varrho \varrho}\right) .
$$

Since $K^{(n)}$ is invariant under $D$ by Lemma 2 ii), the vectors $E_{1} A(\sigma, f) \Omega$ with $\sigma \in K^{(n)}$ generate a subspace $\mathscr{H}_{1}^{(n)} \subset \mathscr{H}_{1}$ which is invariant under $U(a, \Lambda)$. Let $\mathscr{H}_{1}^{\text {irr }}$ be an irreducible subspace of $\mathscr{H}_{1}$. If $U_{1} \mid \mathscr{H}_{1}^{(n)}$ does not contain a subrepresentation that is equivalent to $U_{1} \mid \mathscr{H}_{1}^{\text {irr }}$, then $\mathscr{H}_{1}^{(n)}$ and $\mathscr{H}_{1}^{\text {irr }}$ are orthogonal. Since $\bigcup_{n} \mathscr{H}_{1}^{(n)}$ is dense in $\mathscr{H}_{1}$, this is not possible for all $n$. This proves part ii) of the lemma.

Now let $\Lambda_{\lambda}$ be as in Lemma 1 and put $M_{\sigma \varrho}(\lambda)=M_{\sigma \varrho}\left(\Lambda_{\lambda} \vec{p}\right)$. Since $\Lambda_{\lambda} \bar{p}=\left(\lambda^{2}, 0,0, \lambda^{2}\right)$, this is a polynomial in $\lambda$ by Lemma $\left.2 \mathrm{i}\right)$. Because of Lemma 3 we may even assume that the degree of the polynomials $M_{\sigma \varrho}(\lambda)$ does not exceed some fixed number $2 L$, say, for all $\sigma, \varrho \in K$. In analogy to the construction of $\bar{K}_{1}$ we form Hilbert spaces $\bar{K}_{\lambda}$ by completing the pre-Hilbert spaces $K_{\lambda}=K / N_{\lambda}$, where $N_{\lambda}=\left\{\sigma \mid M_{\sigma \sigma}(\lambda)=0\right\}$ and the scalar product is

$$
\left([\sigma]_{\lambda},[\varrho]_{\lambda}\right)=M_{\sigma \varrho}(\lambda)
$$

with $[\tau]_{\lambda}=\tau+N_{\lambda} . \bar{K}_{\lambda=1}$ is identical to the previously defined $\bar{K}_{1}$.

Since $\mathscr{E}_{2}$ not only leaves $\bar{p}$ invariant, but $\Lambda_{\lambda} \bar{p}=\lambda^{2} \bar{p}$ as well, we have by Lemma 2 ii) a unitary representation $V_{\lambda}$ of $\mathscr{E}_{2}$ on $\bar{K}_{\lambda}$ if we define

$$
V_{\lambda}(\Lambda)[\sigma]_{\lambda}=[D(\Lambda) \sigma]_{\lambda} .
$$

Furthermore, Lemma 2 ii) also implies that the correspondence $[\sigma]_{1} \mapsto\left[D\left(\Lambda_{\lambda}\right) \sigma\right]_{\lambda}$ defines a unitary mapping $\bar{K}_{1} \rightarrow \bar{K}_{\lambda}$. An immediate consequence is the following lemma:

Lemma 4. $V_{1}(\Lambda)$ is unitarily equivalent to $V_{\lambda}\left(\Lambda_{\lambda} \cdot \Lambda \cdot \Lambda_{\lambda}^{-1}\right)$.

As a last preparatory step we embed the spaces $\bar{K}_{\lambda}$ into a common Hilbert space. We define a scalar product on $K$ :

$$
(\sigma, \varrho)=\sum_{v=0}^{2 L} M_{\sigma \varrho}(v) .
$$


It is not necessarily positive definite, but this can be corrected as usual by forming the quotient space $K / N$ with $N=\{\sigma \in K \mid(\sigma, \sigma)=0\}$. We denote the rest classes by $[\tau]$ and the completion of $K / N$ by $\bar{K}$.

If $m_{\sigma} \leqq 2 L$ is the degree of the polynomial $M_{\sigma \sigma}(\lambda)$, we have by the Lagrange interpolation formula

$$
M_{\sigma \sigma}(\lambda)=\sum_{v=0}^{m_{\sigma}} \frac{F_{m_{\sigma}}(\lambda)}{(\lambda-v) F_{m_{\sigma}}^{\prime}(v)} \cdot M_{\sigma \sigma}(v),
$$

where $F_{n}(x)=\prod_{\mu=0}^{n}(x-\mu)$. Because $M_{\sigma \sigma}(\lambda) \geqq 0$ for all $\lambda$, it follows that

$$
0 \leqq M_{\sigma \sigma}(\lambda) \leqq\left(\max _{0 \leqq x \leqq \mu \leqq 2 L} \frac{F_{\mu}(\lambda)}{(\lambda-\chi) F_{\mu}^{\prime}(x)}\right) \cdot \sum_{v=0}^{2 L} M_{\sigma \sigma}(v)=c_{\lambda} \cdot(\sigma, \sigma)
$$

with $c_{\lambda}<\infty$.

It follows that $M_{\sigma e}(\lambda)$ defines a bounded nonnegative sesquilinearform on $\bar{K}$, i.e. there exist bounded, positive semidefinite operators $M_{\lambda}$ on $\bar{K}$ such that

$$
\left([\sigma], M_{\lambda}[\varrho]\right)=M_{\sigma \varrho}(\lambda) .
$$

The matrix elements of $M_{\lambda}$ between states of the form $[\tau]$ are thus polynomials in $\lambda$ of degree $\leqq 2 L$ and $([\sigma],[\varrho])=\sum_{v=0}^{2 L}\left([\sigma], M_{v}[\varrho]\right)$. Since $M_{\lambda}$ is bounded and since the limit of a pointwise convergent sequence of polynomials of degree $\leqq 2 L$ is also a polynomial, this remains true for arbitrary vectors in $\bar{K}$.

Applying Lemma 2 ii) once more, we become a unitary representation $V$ of $\mathscr{E}_{2}$ on $\bar{K}$ such that $V(\Lambda)$ commutes with all $M_{\lambda}$, if we define

$$
V(\Lambda)[\sigma]=[D(\Lambda) \sigma] .
$$

The spaces $\operatorname{Ker} M_{\lambda}$ and their orthogonal complements $\left(\operatorname{Ker} M_{\lambda}\right)^{\perp}$ are invariant under $V(\Lambda)$, and we claim that the restriction $V \mid\left(\operatorname{Ker} M_{\lambda}\right)^{\perp}$ is unitarily equivalent to $V_{\lambda}$ as defined by (6). This is easily seen if we note that since $M_{\lambda}$ is bounded we can use it to define a new scalar product on $\left(\operatorname{Ker} M_{\lambda}\right)^{\perp}:(\varphi, \psi)_{\lambda}=\left(\varphi, M_{\lambda} \psi\right) . V$ remains unitary because $V(\Lambda)$ and $M_{\lambda}$ commute and the mapping $\bar{K}_{\lambda} \rightarrow\left(\operatorname{Ker} M_{\lambda}\right)^{\perp}$ given by

$$
[\sigma]_{\lambda} \mapsto P_{\lambda}[\sigma]
$$

where $P_{\lambda}$ is the projector on $\left(\operatorname{Ker} M_{\lambda}\right)^{\perp}$, is now obviously unitary. This mapping is an intertwining operator between $V$ and $V_{\lambda}$.

Assume now that $\bar{K}_{1}$ contains an irreducible subspace $\bar{K}_{1}^{\text {irr }}$ such that $V_{1} \mid \bar{K}_{1}^{\mathrm{irr}}=V_{\Xi, \alpha}$. If the same is true for $\bar{K}_{\lambda}$, then $V_{1}$ must also contain $V_{\lambda^{2} \Xi, \alpha}$ by Lemmas 1 and 4 . Since inequivalent irreducible representations 
correspond to orthogonal subspaces and because $\bar{K}_{1}$ is separable (otherwise $\mathscr{H}_{1}$ would not be separable) this is only possible for countably many $\lambda . \bar{K}_{1}^{\text {irr }}$ is thus orthogonal to almost all $\left(\operatorname{Ker} M_{\lambda}\right)^{\perp}$, i.e. $\bar{K}_{1}^{\text {irr }} \subset \operatorname{Ker} M_{\lambda}$ for almost every $\lambda$. But $\varphi \in \operatorname{Ker} M_{\lambda}$ means $\left(\varphi, M_{\lambda} \varphi\right)=0$, and since this is continuous in $\lambda$ (even a polynomial), it cannot vanish almost everywhere unless it vanishes identically. Because $(\varphi, \varphi)=\sum_{\nu=0}^{2 L}\left(\varphi, M_{v} \varphi\right)$ we conclude that $\varphi=0$. Therefore, $\bar{K}_{1}^{\mathrm{irr}}=\{0\}$, a contradiction to the hypothesis $V_{1} \mid \bar{K}_{1}^{\text {irr }}=V_{\varepsilon, \alpha}$.

Acknowledgements. The author wishes to thank Professor H. J. Borchers for proposing the problem to him and for many helpful discussions. He also wishes to thank Dr. G. C. Hegerfeldt and Dr. H. Roos for critical comments.

\section{References}

1. Wigner, E. P.: Ann. Math. 40, 149-204 (1939).

2. - Z. Physik 124, 665-684 (1948).

3. McKerrell, A.: Proc. Roy. Soc. London A 285, 287-296 (1965).

4. Weinberg, S.: Phys. Rev. 133B, 1318-1332 (1964) and Phys. Rev. 134B, 882-896 (1964).

5. Streater, R. F., Wightman, A. S.: PCT, Spin and Statistics and all that. New York: W. A. Benjamin 1964.

6. Bros, J., Epstein, H., Glaser, V.: Commun. Math. Phys. 6, 77-100 (1967).

7. Strocchi, F.: Phys. Rev. 166, 1302-1307 (1969).

Jakob Yngvason

Institut f. Theoretische Physik der Universität D-3400 Göttingen 